\title{
STUDIES OF PULMONARY HYPERTENSION. IX. THE EFFECTS OF INTRAVENOUS HEXAMETHONIUM ON PULMONARY CIRCULATION IN PATIENTS WITH MITRAL STENOSIS ${ }^{1}$
}

\author{
By PAUL N. YU, R. E. NYE, JR., ${ }^{2}$ FRANK W. LOVEJOY, JR., BERNARD F. SCHREINER, ${ }^{8}$ \\ AND BERNARD J. B. YIM 4 \\ (From the Chest Laboratory of the Department of Medicine, University of Rochester School of \\ Medicine and Dentistry, and Medical Clinics, Strong Memorial and Rochester \\ Municipal Hospitals, Rochester, N. Y.)
}

(Submitted for publication August 29, 1957; accepted October 17, 1957)

During the past five years, circulatory effects of intravenous hexamethonium have been studied in normal subjects and in patients with various cardiac diseases (1-10). With adequate doses of the drug a significant reduction of the systemic blood pressure was observed in almost all cases. In many patients, particularly those with pulmonary hypertension, a reduction of the pulmonary artery pressure was also seen. However, the pulmonary "capillary" or pulmonary artery wedge pressures were recorded in only a small number of cases and changes of resistances in the pulmonary circuit were seldom studied.

The purposes of this paper are: 1 ) to report the hemodynamic effects of hexamethonium in a series of patients with mitral stenosis, 2) to discuss the probable mechanism of the changes of the resistances and pressures in the pulmonary circuit of these patients, and 3) to stress the therapeutic benefit of hexamethonium in patients with acute pulmonary edema secondary to mitral stenosis.

\section{CLINICAL MATERIAL AND METHOD}

Twenty-seven patients with predominant mitral stenosis were studied. There were 10 males and 17 females. Their ages ranged from 13 to 58 years. None of the patients had a significant degree of mitral insufficiency, a demonstrable lesion of other valves, or systemic hypertension.

1 This study was supported in part by a grant-in-aid (H 222 C7) from the National Heart Institute of the National Institutes of Health, by a grant from the American Heart Association and New York Heart Assembly, and by the Hochstetter Fund and Ernest L. Woodward Fund.

2 Present address: Department of Physiology, Dartmouth Medical School, Hanover, N. H.

3 Postdoctoral Research Fellow, National Heart Institute, United States Public Health Service (1956-1958).

4 Trainee, National Heart Institute, United States Public Health Service (1956-1957).
The patients were studied two to three hours after a light breakfast. In all cases 100 to $200 \mathrm{mg}$. of methyprylon (Noludar (\$) ${ }^{5}$ was given by mouth as premedication.

The methods of determining the cardiac output and recording blood pressures have been described in detail in several previous papers (11-14). The formulae used to derive the resistances and ventricular work against pressures and mitral valve flow were adopted from the papers by Gorlin and his co-workers $(15,16)$. The mean pressures were measured by planimetric integration. Pulmonary "capillary" pressure was substituted for pulmonary venous and left atrial pressures. Pulmonary vascular resistance is the resistance between pulmonary artery and pulmonary vein. Total pulmonary resistance is the resistance between pulmonary artery and a hypothetical sink at atmospheric pressure. The difference between total pulmonary resistance and pulmonary vascular resistance is termed "left heart resistance" instead of "mitral valve resistance" $(17,18)$. It includes the resistance at the mitral valve as well as the resistance to filling offered by the left ventricle in diastole. In patients with pure mitral stenosis, however, most of the calculated value is ascribable to resistance at the valve itself.

The right ventricular diastolic pressure was used in calculating the right ventricular work against pressure. In most cases right ventricular diastolic pressure was only recorded after hexamethonium administration and this was used for calculation of the work both before and after the drug. In several cases the control right ventricular diastolic pressure was little different from the value after injection. It was felt that the error would be small in calculating right ventricular work against pressure by using only the value following injection.

In calculating left ventricular work against pressure, the left ventricular diastolic pressure was assumed to be $5 \mathrm{~mm}$. $\mathrm{Hg}$ in each case, because no left ventricular pressure was obtained at the time of cardiac catheterization. The calculation was discarded in five patients whose left ventricular diastolic pressure was later found, at operation, to exceed $8 \mathrm{~mm}$. $\mathrm{Hg}$.

After control measurements of cardiac output and femoral and pulmonary artery pressures (in all patients) and of pulmonary "capillary" pressure (in all but three

\footnotetext{
${ }^{5}$ Courtesy of Hoffman LaRoche Company, Nutley, N. J.
} 
TABLE I

Hemodynamic data in patients with mitral stenosis before and after intravenous injection of hexamethonium *

\begin{tabular}{|c|c|c|c|c|c|c|c|c|c|c|c|}
\hline \multirow[b]{2}{*}{ Case No. } & \multirow{2}{*}{\multicolumn{2}{|c|}{${ }_{\text {BSA }}^{\text {Age }}$}} & \multirow{2}{*}{$\begin{array}{c}\text { Dosage } \\
\text { of } \mathrm{C}_{6} \\
(\mathrm{mg.})\end{array}$} & \multirow{2}{*}{$\begin{array}{c}\text { Periods } \\
\text { of } \\
\text { study }\end{array}$} & \multirow[b]{2}{*}{ R.Q. } & \multicolumn{5}{|c|}{ Blood flow } & \multirow{2}{*}{$\begin{array}{c}\mathrm{O}_{2} \\
\text { saturation } \\
(\%) \\
\end{array}$} \\
\hline & & & & & & $\begin{array}{c}\mathrm{O}_{2} \\
\text { cons. }\end{array}$ & $\begin{array}{l}\text { A-V O } \\
\text { diff. }\end{array}$ & C.I. & H.R. & S.I. & \\
\hline 1. J. K. & 34 & $2.00^{M}$ & 12 & II (25) & $\begin{array}{l}0.86 \\
0.85\end{array}$ & $\begin{array}{l}144 \\
143\end{array}$ & $\begin{array}{l}41.5 \\
41.4\end{array}$ & $\begin{array}{l}3.47 \\
3.46\end{array}$ & $\begin{array}{r}90 \\
100\end{array}$ & $\begin{array}{l}39 \\
34\end{array}$ & $\begin{array}{l}92.9 \\
92.7\end{array}$ \\
\hline 2. R. B. & 55 & $1.57^{\mathrm{F}}$ & 5 & II (16) & $\begin{array}{l}0.82 \\
0.75\end{array}$ & $\begin{array}{l}157 \\
134\end{array}$ & $\begin{array}{l}39.6 \\
37.8\end{array}$ & $\begin{array}{l}3.96 \\
3.54\end{array}$ & $\begin{array}{l}125 \\
108\end{array}$ & $\begin{array}{l}32 \\
33\end{array}$ & $\begin{array}{l}90.2 \\
89.6\end{array}$ \\
\hline 3. C. W. & 56 & $2.10^{M}$ & 6 & II $\underset{(22)}{\text { II }}$ & $\begin{array}{l}0.87 \\
0.92 \\
0.87\end{array}$ & $\begin{array}{l}165 \\
146 \\
164\end{array}$ & $\begin{array}{l}55.4 \\
56.0 \\
52.5\end{array}$ & $\begin{array}{l}2.97 \\
2.61 \\
3.12\end{array}$ & $\begin{array}{l}80 \\
82 \\
88\end{array}$ & $\begin{array}{l}37 \\
32 \\
35\end{array}$ & $\begin{array}{l}96.1 \\
98.3 \\
95.8\end{array}$ \\
\hline 4. J. P. & 36 & ${ }_{1.63^{F}}$ & 10 & $\begin{array}{l}\text { I } \\
\text { II (33) } \\
\quad(80)\end{array}$ & $\begin{array}{l}0.80 \\
0.78 \\
0.79\end{array}$ & $\begin{array}{l}109 \\
128 \\
104\end{array}$ & $\begin{array}{l}51.1 \\
51.1 \\
50.6\end{array}$ & $\begin{array}{l}2.13 \\
2.50 \\
2.05\end{array}$ & $\begin{array}{l}67 \\
70 \\
48\end{array}$ & $\begin{array}{l}32 \\
36 \\
43\end{array}$ & $\begin{array}{l}94.1 \\
96.2 \\
91.6\end{array}$ \\
\hline 5. G. W. & 30 & $1.60^{\mathrm{F}}$ & 6 & II $(31)$ & $\begin{array}{l}0.84 \\
0.78\end{array}$ & $\begin{array}{l}128 \\
132\end{array}$ & $\begin{array}{l}38.0 \\
38.9\end{array}$ & $\begin{array}{l}3.37 \\
3.39\end{array}$ & $\begin{array}{l}118 \\
126\end{array}$ & $\begin{array}{l}29 \\
27\end{array}$ & $\begin{array}{l}99.6 \\
92.3\end{array}$ \\
\hline 6. A.W. & 48 & $1.90^{\mathrm{M}}$ & 9 & II (32) & $\begin{array}{l}0.87 \\
0.82\end{array}$ & $\begin{array}{l}128 \\
131\end{array}$ & $\begin{array}{l}48.6 \\
44.5\end{array}$ & $\begin{array}{l}2.64 \\
2.95\end{array}$ & $\begin{array}{l}81 \\
90\end{array}$ & $\begin{array}{l}33 \\
33\end{array}$ & $\begin{array}{l}90.6 \\
91.7\end{array}$ \\
\hline 7. D. C. & 13 & $1.39^{\mathrm{F}}$ & 6 & II (29) & $\begin{array}{l}0.89 \\
0.88\end{array}$ & $\begin{array}{l}134 \\
144\end{array}$ & $\begin{array}{l}44.3 \\
42.2\end{array}$ & $\begin{array}{l}3.02 \\
3.41\end{array}$ & $\begin{array}{l}103 \\
106\end{array}$ & $\begin{array}{l}29 \\
32\end{array}$ & $\begin{array}{l}98.0 \\
95.0\end{array}$ \\
\hline 8. M. B. & 17 & $1.90^{M}$ & 7 & II (25) & & 159 & $\begin{array}{l}51.2 \\
50.6\end{array}$ & 3.10 & $\begin{array}{r}93 \\
106\end{array}$ & 34 & $\begin{array}{l}98.0 \\
95.0\end{array}$ \\
\hline 9. S. M. & 40 & $1.51^{\mathrm{F}}$ & 10 & $\begin{array}{l}\text { I } \\
\text { II (23) } \\
\quad(41)\end{array}$ & $\begin{array}{l}0.80 \\
0.88 \\
0.90\end{array}$ & $\begin{array}{l}129 \\
131 \\
125\end{array}$ & $\begin{array}{l}53.2 \\
53.8 \\
53.5\end{array}$ & $\begin{array}{l}2.40 \\
2.40 \\
2.30\end{array}$ & $\begin{array}{r}90 \\
100 \\
75\end{array}$ & $\begin{array}{l}27 \\
24 \\
31\end{array}$ & $\begin{array}{l}94.3 \\
95.0 \\
93.7\end{array}$ \\
\hline 10. J.S. & 26 & $1.64^{M}$ & 9 & $\begin{array}{l}\text { I } \\
\text { II (25) } \\
(44)\end{array}$ & $\begin{array}{l}0.83 \\
0.85 \\
0.74\end{array}$ & $\begin{array}{l}151 \\
138 \\
147\end{array}$ & $\begin{array}{l}55.4 \\
49.8 \\
45.6\end{array}$ & $\begin{array}{l}2.72 \\
2.79 \\
3.22\end{array}$ & $\begin{array}{l}92 \\
88 \\
83\end{array}$ & $\begin{array}{l}30 \\
32 \\
39\end{array}$ & $\begin{array}{l}91.6 \\
94.6 \\
92.6\end{array}$ \\
\hline 11. N. M. & 41 & ${ }_{1.78^{M}}$ & 9 & II $\underset{(73)}{(31)}$ & $\begin{array}{l}0.83 \\
0.85 \\
0.80\end{array}$ & $\begin{array}{l}150 \\
134 \\
130\end{array}$ & $\begin{array}{l}62.7 \\
66.5 \\
66.1\end{array}$ & $\begin{array}{l}2.39 \\
2.02 \\
1.97\end{array}$ & $\begin{array}{r}80 \\
100 \\
90\end{array}$ & $\begin{array}{l}30 \\
20 \\
22\end{array}$ & $\begin{array}{l}96.2 \\
96.1 \\
95.8\end{array}$ \\
\hline 12. G. P. & 38 & $1.51^{F}$ & 14 & II (45) & & $\begin{array}{l}112 \\
107\end{array}$ & $\begin{array}{l}52.6 \\
53.6\end{array}$ & $\begin{array}{l}2.15 \\
2.00\end{array}$ & $\begin{array}{l}88 \\
86\end{array}$ & $\begin{array}{l}24 \\
23\end{array}$ & $\begin{array}{l}99.5 \\
94.1\end{array}$ \\
\hline 13. M. S. & 24 & $1.40^{\mathrm{F}}$ & 2 & II (29) & $\begin{array}{l}0.81 \\
0.83\end{array}$ & $\begin{array}{l}132 \\
138\end{array}$ & $\begin{array}{l}64.6 \\
65.9\end{array}$ & $\begin{array}{l}2.05 \\
2.09\end{array}$ & $\begin{array}{r}95 \\
108\end{array}$ & $\begin{array}{l}22 \\
19\end{array}$ & $\begin{array}{l}96.4 \\
94.3\end{array}$ \\
\hline 14. M. F. & 58 & $1.61^{\mathrm{F}}$ & 8 & $\begin{array}{l}\text { I } \\
\text { II (24) } \\
\quad(49)\end{array}$ & $\begin{array}{l}0.77 \\
0.85 \\
0.89\end{array}$ & $\begin{array}{l}137 \\
118 \\
124\end{array}$ & $\begin{array}{l}74.3 \\
53.9 \\
57.3\end{array}$ & $\begin{array}{l}1.85 \\
2.19 \\
2.16\end{array}$ & $\begin{array}{l}69 \\
74 \\
63\end{array}$ & $\begin{array}{l}27 \\
30 \\
34\end{array}$ & $\begin{array}{l}94.2 \\
90.2 \\
90.4\end{array}$ \\
\hline 15. K. K. & 37 & $1.67^{\mathrm{F}}$ & 22.5 & II (27) & & $\begin{array}{l}144 \\
125 \\
121\end{array}$ & $\begin{array}{l}59.0 \\
54.1 \\
56.0\end{array}$ & $\begin{array}{l}2.44 \\
2.30 \\
2.16\end{array}$ & $\begin{array}{l}90 \\
90 \\
84\end{array}$ & $\begin{array}{l}27 \\
26 \\
26\end{array}$ & $\begin{array}{l}91.7 \\
89.2 \\
90.5\end{array}$ \\
\hline 16. A.F. & 47 & $1.66^{\mathrm{M}}$ & 8 & $\begin{array}{l}\text { I } \\
\text { II (23) } \\
\quad(72)\end{array}$ & $\begin{array}{l}0.81 \\
0.83 \\
0.80\end{array}$ & $\begin{array}{l}125 \\
125 \\
135\end{array}$ & $\begin{array}{l}72.0 \\
74.7 \\
77.1\end{array}$ & $\begin{array}{l}1.70 \\
1.70 \\
1.80\end{array}$ & $\begin{array}{l}73 \\
97 \\
87\end{array}$ & $\begin{array}{l}24 \\
17 \\
20\end{array}$ & $\begin{array}{l}90.0 \\
89.0 \\
89.0\end{array}$ \\
\hline 17. E. W. & 30 & $1.55^{F}$ & 18 & II (34) & & $\begin{array}{l}131 \\
145\end{array}$ & $\begin{array}{l}68.1 \\
60.0\end{array}$ & $\begin{array}{l}1.93 \\
2.42\end{array}$ & $\begin{array}{l}94 \\
94\end{array}$ & $\begin{array}{l}21 \\
26\end{array}$ & $\begin{array}{l}91.1 \\
88.4\end{array}$ \\
\hline 18. A. K. & 36 & $1.40^{F}$ & 6 & II $\underset{(42)}{(15)}$ & $\begin{array}{l}0.85 \\
0.85 \\
0.90\end{array}$ & $\begin{array}{l}128 \\
136 \\
128\end{array}$ & $\begin{array}{l}57.0 \\
53.1 \\
58.7\end{array}$ & $\begin{array}{l}2.24 \\
2.56 \\
2.18\end{array}$ & $\begin{array}{l}88 \\
88 \\
71\end{array}$ & $\begin{array}{l}25 \\
29 \\
30\end{array}$ & $\begin{array}{l}96.4 \\
92.7 \\
90.8\end{array}$ \\
\hline 19. R. K. & 53 & $1.80^{M}$ & 15 & II (30) & $\begin{array}{l}0.88 \\
0.90\end{array}$ & $\begin{array}{l}116 \\
112\end{array}$ & $\begin{array}{l}75.7 \\
60.6\end{array}$ & $\begin{array}{l}1.53 \\
1.85\end{array}$ & $\begin{array}{l}66 \\
70\end{array}$ & $\begin{array}{l}23 \\
26\end{array}$ & $\begin{array}{l}99.3 \\
95.9\end{array}$ \\
\hline 20. H. J. & 41 & $1.48^{\mathrm{F}}$ & 14 & II (50) & $\begin{array}{l}0.84 \\
0.91\end{array}$ & $\begin{array}{l}158 \\
131\end{array}$ & $\begin{array}{l}73.4 \\
50.0\end{array}$ & $\begin{array}{l}2.15 \\
2.62\end{array}$ & $\begin{array}{l}80 \\
69\end{array}$ & $\begin{array}{l}27 \\
38\end{array}$ & $\begin{array}{l}92.9 \\
90.2\end{array}$ \\
\hline 21. E. J. & 47 & $1.44^{F}$ & 7 & II $\begin{array}{l}\text { I (28) } \\
\text { (64) }\end{array}$ & $\begin{array}{l}0.95 \\
0.90 \\
0.85\end{array}$ & $\begin{array}{r}113 \\
96 \\
112\end{array}$ & $\begin{array}{l}77.4 \\
58.2 \\
62.7\end{array}$ & $\begin{array}{l}1.46 \\
1.65 \\
1.78\end{array}$ & $\begin{array}{l}92 \\
88 \\
78\end{array}$ & $\begin{array}{l}16 \\
19 \\
23\end{array}$ & $\begin{array}{l}99.7 \\
93.3 \\
93.0\end{array}$ \\
\hline 22. B. K. & 34 & $1.72^{F}$ & 6 & II (30) & & $\begin{array}{r}118 \\
94\end{array}$ & $\begin{array}{l}99.1 \\
79.6\end{array}$ & $\begin{array}{l}1.19 \\
1.19\end{array}$ & $\begin{array}{r}102 \\
78\end{array}$ & $\begin{array}{l}12 \\
15\end{array}$ & $\begin{array}{l}89.8 \\
84.4\end{array}$ \\
\hline 23. A. C. & 47 & $1.86^{\mathrm{M}}$ & 10 & II $(47)$ & $\begin{array}{l}0.88 \\
0.91\end{array}$ & $\begin{array}{l}164 \\
149\end{array}$ & $\begin{array}{l}65.7 \\
54.5\end{array}$ & $\begin{array}{l}2.45 \\
2.16\end{array}$ & $\begin{array}{l}74 \\
84\end{array}$ & $\begin{array}{l}33 \\
26\end{array}$ & $\begin{array}{l}91.3 \\
90.6\end{array}$ \\
\hline 24. M. Mc. & 55 & $1.49^{\mathrm{F}}$ & 14 & II (39) & $\begin{array}{l}1.03 \\
0.77\end{array}$ & $\begin{array}{l}115 \\
105\end{array}$ & $\begin{array}{l}52.6 \\
60.6\end{array}$ & $\begin{array}{l}2.19 \\
1.74\end{array}$ & $\begin{array}{r}96 \\
107\end{array}$ & $\begin{array}{l}23 \\
16\end{array}$ & $\begin{array}{l}97.0 \\
98.0\end{array}$ \\
\hline 25. G. A. & 39 & ${ }_{1.51}^{\mathrm{F}}$ & 11 & II (29) & & $\begin{array}{l}129 \\
113\end{array}$ & $\begin{array}{l}59.7 \\
55.5\end{array}$ & $\begin{array}{l}2.16 \\
2.04\end{array}$ & $\begin{array}{l}82 \\
81\end{array}$ & $\begin{array}{l}27 \\
25\end{array}$ & $\begin{array}{l}99.2 \\
95.2\end{array}$ \\
\hline 26. D. R. & 42 & $1.86^{M}$ & 9 & $\begin{array}{l}\text { I } \\
\text { II (29) } \\
\text { (48) }\end{array}$ & $\begin{array}{l}0.96 \\
0.97\end{array}$ & $\begin{array}{l}109 \\
110 \\
113\end{array}$ & $\begin{array}{l}37.4 \\
55.3 \\
48.6\end{array}$ & $\begin{array}{l}3.34 \\
2.16 \\
2.35\end{array}$ & $\begin{array}{l}84 \\
80 \\
85\end{array}$ & $\begin{array}{l}40 \\
27 \\
28\end{array}$ & $\begin{array}{l}91.8 \\
94.0 \\
92.0\end{array}$ \\
\hline 27. J. Sc. & 40 & ${ }_{1.66}^{F}$ & 12 & II (35) & $\begin{array}{l}0.88 \\
0.80\end{array}$ & $\begin{array}{l}120 \\
144\end{array}$ & $\begin{array}{l}51.6 \\
44.7\end{array}$ & $\begin{array}{l}2.33 \\
3.22\end{array}$ & $\begin{array}{l}98 \\
98\end{array}$ & $\begin{array}{l}23 \\
32\end{array}$ & $\begin{array}{l}90.9 \\
90.9\end{array}$ \\
\hline
\end{tabular}

*BSA = Body surface area $\left(M^{2}{ }^{2}\right)$

$\mathrm{C}_{\mathrm{B}}=$ Hexamethonium ion

II = Control period. $\mathrm{O}_{2}$ cons. $=$ Oxygen consumption $(\mathrm{ml} . / \mathrm{M} .2 / \mathrm{min} . \mathrm{STPD})$ the minute after the start of injection of $C_{-6}$. $\mathrm{A}-\mathrm{V} \mathrm{O}_{2}$ diff. = Arteriovenous oxygen difference (mi./L.) R.Q. = Respiratory quotient. 
TABLE I-Continued

\begin{tabular}{|c|c|c|c|c|c|c|c|c|c|c|c|c|c|c|c|c|}
\hline \multirow[b]{3}{*}{ Case No. } & \multirow{3}{*}{$\begin{array}{l}\text { Periods } \\
\text { of } \\
\text { study }\end{array}$} & \multicolumn{9}{|c|}{ Pressures $(\mathrm{mm} . \mathrm{Hg}$ ) } & \multicolumn{4}{|c|}{$\begin{array}{c}\text { Resistances } \\
\left.\text { (dynes sec./cm. }{ }^{5}\right)\end{array}$} & \multicolumn{2}{|c|}{$\begin{array}{c}\text { Ventricular } \\
\text { work against } \\
\text { ppressure }\end{array}$} \\
\hline & & \multicolumn{2}{|c|}{ F.A. } & \multicolumn{2}{|l|}{ P.A. } & \multirow{2}{*}{$\begin{array}{l}\text { "P.C." } \\
(\mathbf{M})\end{array}$} & \multirow{2}{*}{$\begin{array}{l}\text { P.A.-. } \\
\text { "P.C." } \\
\text { (M) }\end{array}$} & \multirow{2}{*}{$\begin{array}{l}\text { R.V. } \\
(\mathbf{S} / \mathbf{D})\end{array}$} & \multirow{2}{*}{ R.A. } & \multirow{2}{*}{ L.V. } & \multirow[b]{2}{*}{ TSR } & \multirow[b]{2}{*}{ TPR } & \multirow[b]{2}{*}{ PVR } & \multirow[b]{2}{*}{ "IJHR" } & \multirow{2}{*}{\multicolumn{2}{|c|}{$\begin{array}{c}\text { Left } \\
\left(\mathrm{Kg}_{\mathbf{g}} \mathbf{M} . / \mathbf{M} . .^{2}\right)\end{array}$}} \\
\hline & & (S/D & M) & $(\mathrm{S} / \mathrm{D}$ & M) & & & & & & & & & & & \\
\hline 1. J. K. & II (25) & $\begin{array}{l}102 / 63 \\
95 / 59\end{array}$ & $\begin{array}{l}74 \\
72\end{array}$ & $\begin{array}{l}50 / 28 \\
46 / 24\end{array}$ & $\begin{array}{l}36 \\
34\end{array}$ & $\begin{array}{l}29 \\
28\end{array}$ & $\begin{array}{l}7 \\
6\end{array}$ & $62 / 6$ & 5 & 14 & $\begin{array}{l}830 \\
840\end{array}$ & $\begin{array}{l}410 \\
380\end{array}$ & $\begin{array}{l}80 \\
60\end{array}$ & $\begin{array}{l}330 \\
320\end{array}$ & & $\begin{array}{l}1.5 \\
1.4\end{array}$ \\
\hline 2. R. B. & II (16) & $\begin{array}{l}166 / 74 \\
127 / 66\end{array}$ & $\begin{array}{r}104 \\
87\end{array}$ & $\begin{array}{l}47 / 29 \\
34 / 21\end{array}$ & $\begin{array}{l}35 \\
24\end{array}$ & $\begin{array}{l}29 \\
16\end{array}$ & $\begin{array}{l}6 \\
8\end{array}$ & $43 / 3$ & 1 & 10 & $\begin{array}{l}1,320 \\
1,230\end{array}$ & $\begin{array}{l}440 \\
340\end{array}$ & $\begin{array}{r}80 \\
110\end{array}$ & $\begin{array}{l}360 \\
220\end{array}$ & & $\begin{array}{l}1.8 \\
1.1\end{array}$ \\
\hline 3. C. W. & II (22) & $\begin{array}{l}180 / 90 \\
103 / 49 \\
109 / 64\end{array}$ & $\begin{array}{r}118 \\
65 \\
81\end{array}$ & $\begin{array}{l}73 / 30 \\
36 / 19 \\
44 / 26\end{array}$ & $\begin{array}{l}43 \\
25 \\
32\end{array}$ & $\begin{array}{l}28 \\
13\end{array}$ & $\begin{array}{l}15 \\
12\end{array}$ & $\begin{array}{l}70 / 7 \\
44 / 5\end{array}$ & 5 & 6 & $\begin{array}{r}1,490 \\
930 \\
970\end{array}$ & $\begin{array}{l}540 \\
360 \\
390\end{array}$ & $\begin{array}{l}190 \\
170\end{array}$ & $\begin{array}{l}350 \\
190\end{array}$ & $\begin{array}{l}4.8 \\
2.2 \\
3.3\end{array}$ & $\begin{array}{l}1.5 \\
0.7 \\
1.2\end{array}$ \\
\hline 4. J. P. & II $\underset{(83)}{\text { I }}$ & $\begin{array}{r}102 / 52 \\
83 / 36 \\
86 / 38\end{array}$ & $\begin{array}{l}71 \\
58 \\
48\end{array}$ & $\begin{array}{l}40 / 18 \\
26 / 15 \\
28 / 12\end{array}$ & $\begin{array}{l}25 \\
19 \\
16\end{array}$ & 18 & 7 & $30 / 6$ & 4 & 6 & $\begin{array}{l}1,610 \\
1,120 \\
1,110\end{array}$ & $\begin{array}{l}570 \\
370 \\
380\end{array}$ & 160 & 410 & $\begin{array}{l}2.0 \\
1.9 \\
1.3\end{array}$ & $\begin{array}{l}0.6 \\
0.5 \\
0.3\end{array}$ \\
\hline 5. G. W. & II (31) & $104 / 73$ & 87 & $\begin{array}{l}66 / 40 \\
59 / 36\end{array}$ & $\begin{array}{l}47 \\
41\end{array}$ & $\begin{array}{l}37 \\
27\end{array}$ & $\begin{array}{l}10 \\
14\end{array}$ & $57 / 4$ & 4 & 8 & $\begin{array}{l}1,130 \\
1,100\end{array}$ & $\begin{array}{l}640 \\
610\end{array}$ & $\begin{array}{l}240 \\
210\end{array}$ & $\begin{array}{l}400 \\
400\end{array}$ & 4.0 & $\begin{array}{l}2.1 \\
1.8\end{array}$ \\
\hline 6. A. W. & II (32) & $\begin{array}{l}148 / 76 \\
124 / 76\end{array}$ & $\begin{array}{r}102 \\
93\end{array}$ & $\begin{array}{l}65 / 34 \\
62 / 32\end{array}$ & $\begin{array}{l}46 \\
42\end{array}$ & $\begin{array}{l}38 \\
29\end{array}$ & $\begin{array}{r}8 \\
13\end{array}$ & $65 / 11$ & 10 & 13 & $\begin{array}{l}1,620 \\
1,330\end{array}$ & $\begin{array}{l}730 \\
600\end{array}$ & $\begin{array}{l}220 \\
190\end{array}$ & $\begin{array}{l}510 \\
410\end{array}$ & & $\begin{array}{l}1.4 \\
1.3\end{array}$ \\
\hline 7. D. C. & II (29) & $\begin{array}{l}92 / 60 \\
96 / 60\end{array}$ & $\begin{array}{l}72 \\
75\end{array}$ & $\begin{array}{l}60 / 30 \\
55 / 35\end{array}$ & $\begin{array}{l}42 \\
43\end{array}$ & $\begin{array}{l}29 \\
36\end{array}$ & $\begin{array}{r}13 \\
7\end{array}$ & $55 / 5$ & 5 & 1 & $\begin{array}{l}1,350 \\
1,250\end{array}$ & $\begin{array}{l}790 \\
710\end{array}$ & $\begin{array}{l}240 \\
120\end{array}$ & $\begin{array}{l}550 \\
590\end{array}$ & $\begin{array}{l}2.9 \\
3.4\end{array}$ & $\begin{array}{l}1.3 \\
1.8\end{array}$ \\
\hline 8. M. B. & II $(25)$ & $\begin{array}{r}105 / 61 \\
84 / 60\end{array}$ & $\begin{array}{l}74 \\
66\end{array}$ & $\begin{array}{l}80 / 46 \\
67 / 40\end{array}$ & $\begin{array}{l}60 \\
49\end{array}$ & $\begin{array}{l}30 \\
\mathbf{3 0}\end{array}$ & $\begin{array}{l}30 \\
19\end{array}$ & $\begin{array}{l}75 / 8 \\
67 / 9\end{array}$ & $\begin{array}{l}7 \\
7\end{array}$ & & 1,010 & 820 & 410 & 410 & 3.0 & 2.3 \\
\hline 9. S. M. & II (23) & $\begin{array}{r}120 / 71 \\
87 / 50 \\
99 / 55\end{array}$ & $\begin{array}{l}89 \\
63 \\
71\end{array}$ & $\begin{array}{l}66 / 29 \\
45 / 23 \\
37 / 14\end{array}$ & $\begin{array}{l}41 \\
32 \\
25\end{array}$ & $\begin{array}{l}26 \\
16 \\
11\end{array}$ & $\begin{array}{l}15 \\
16 \\
14\end{array}$ & $37 / 4$ & 2 & $\mathbf{0}$ & $\begin{array}{l}1,910 \\
1,350 \\
1,630\end{array}$ & $\begin{array}{l}880 \\
690 \\
570\end{array}$ & $\begin{array}{l}320 \\
340 \\
320\end{array}$ & $\begin{array}{l}560 \\
340 \\
250\end{array}$ & $\begin{array}{l}2.9 \\
2.0 \\
2.0\end{array}$ & $\begin{array}{l}1.3 \\
1.0 \\
0.8\end{array}$ \\
\hline 10. J.S. & $\begin{array}{l}\text { I } \\
\text { II (25) } \\
\text { (44) }\end{array}$ & $\begin{array}{l}133 / 83 \\
100 / 60 \\
110 / 61\end{array}$ & $\begin{array}{r}100 \\
76 \\
77\end{array}$ & $\begin{array}{l}80 / 37 \\
69 / 35 \\
56 / 27\end{array}$ & $\begin{array}{l}55 \\
47 \\
38\end{array}$ & $\begin{array}{l}33 \\
30 \\
20\end{array}$ & $\begin{array}{l}22 \\
17 \\
18\end{array}$ & $50 / 3$ & 2 & 6 & $\begin{array}{l}1,760 \\
1,310 \\
1,150\end{array}$ & $\begin{array}{l}950 \\
810 \\
570\end{array}$ & $\begin{array}{l}390 \\
290 \\
270\end{array}$ & $\begin{array}{l}560 \\
520 \\
300\end{array}$ & $\begin{array}{l}3.7 \\
2.8 \\
3.3\end{array}$ & $\begin{array}{l}2.0 \\
1.7 \\
1.6\end{array}$ \\
\hline 11. N. M. & II (31) & $\begin{array}{r}116 / 62 \\
87 / 53 \\
92 / 59\end{array}$ & $\begin{array}{l}80 \\
65 \\
68\end{array}$ & $\begin{array}{l}87 / 38 \\
66 / 39 \\
80 / 38\end{array}$ & $\begin{array}{l}51 \\
48 \\
50\end{array}$ & 25 & 26 & $67 / 7$ & 7 & 7 & $\begin{array}{l}1,670 \\
1,440 \\
1,550\end{array}$ & $\begin{array}{r}960 \\
1,070 \\
1,140\end{array}$ & 490 & 470 & $\begin{array}{l}2.6 \\
1.8 \\
1.8\end{array}$ & $\begin{array}{l}1.6 \\
1.2 \\
1.2\end{array}$ \\
\hline 12. G. P. & II (45) & $\begin{array}{l}148 / 87 \\
160 / 91\end{array}$ & $\begin{array}{l}102 \\
115\end{array}$ & $\begin{array}{l}52 / 30 \\
40 / 25\end{array}$ & $\begin{array}{l}36 \\
30\end{array}$ & $\begin{array}{l}24 \\
18\end{array}$ & $\begin{array}{l}12 \\
13\end{array}$ & $43 / 3$ & 5 & 3 & $\begin{array}{l}2,470 \\
3,000\end{array}$ & $\begin{array}{r}1,010 \\
810\end{array}$ & $\begin{array}{l}320 \\
340\end{array}$ & $\begin{array}{l}690 \\
470\end{array}$ & $\begin{array}{l}3.0 \\
3.1\end{array}$ & $\begin{array}{l}1.0 \\
0.7\end{array}$ \\
\hline 13. M. S. & II (29) & $\begin{array}{r}119 / 76 \\
98 / 61\end{array}$ & $\begin{array}{l}90 \\
72\end{array}$ & $\begin{array}{l}61 / 34 \\
57 / 32\end{array}$ & $\begin{array}{l}46 \\
45\end{array}$ & & & $\begin{array}{l}61 / 9 \\
52 / 7\end{array}$ & $\begin{array}{l}7 \\
6\end{array}$ & 9 & $\begin{array}{l}2,560 \\
2,150\end{array}$ & $\begin{array}{l}1,280 \\
1,230\end{array}$ & & & & $\begin{array}{l}1.1 \\
1.2\end{array}$ \\
\hline 14. M. F. & II (24) & $\begin{array}{l}163 / 85 \\
95 / 52 \\
104 / 53\end{array}$ & $\begin{array}{r}114 \\
63 \\
69\end{array}$ & $\begin{array}{l}80 / 28 \\
72 / 28 \\
70 / 25\end{array}$ & $\begin{array}{l}44 \\
43 \\
40\end{array}$ & $\begin{array}{l}21 \\
17 \\
18\end{array}$ & $\begin{array}{l}23 \\
26 \\
22\end{array}$ & $68 / 11$ & 10 & 16 & $\begin{array}{l}3,000 \\
1,430 \\
1,580\end{array}$ & $\begin{array}{r}1,310 \\
970 \\
920\end{array}$ & $\begin{array}{l}750 \\
590 \\
500\end{array}$ & $\begin{array}{l}560 \\
380 \\
420\end{array}$ & & $\begin{array}{l}0.9 \\
1.0 \\
0.9\end{array}$ \\
\hline 15. K. K. & II (27) & $\begin{array}{r}110 / 74 \\
103 / 62 \\
96 / 58\end{array}$ & $\begin{array}{l}91 \\
76 \\
72\end{array}$ & $\begin{array}{r}127 / 43 \\
104 / 38 \\
94 / 36\end{array}$ & $\begin{array}{l}73 \\
59 \\
56\end{array}$ & $\begin{array}{l}36 \\
26 \\
22\end{array}$ & $\begin{array}{l}37 \\
33 \\
34\end{array}$ & $81 / 4$ & 4 & 2 & $\begin{array}{l}1,780 \\
1,570 \\
1,590\end{array}$ & $\begin{array}{l}1,430 \\
1,210 \\
1,240\end{array}$ & $\begin{array}{l}720 \\
680 \\
750\end{array}$ & $\begin{array}{l}710 \\
530 \\
490\end{array}$ & $\begin{array}{l}3.0 \\
2.3 \\
2.1\end{array}$ & $\begin{array}{l}2.4 \\
1.8 \\
1.6\end{array}$ \\
\hline 16. A. F. & $\begin{array}{l}\text { I } \\
\text { II (23) } \\
\text { (72) }\end{array}$ & $\begin{array}{r}120 / 57 \\
82 / 52 \\
98 / 54\end{array}$ & $\begin{array}{l}81 \\
63 \\
70\end{array}$ & $\begin{array}{l}80 / 36 \\
70 / 38 \\
82 / 42\end{array}$ & $\begin{array}{l}53 \\
49 \\
56\end{array}$ & $\begin{array}{l}31 \\
23 \\
26\end{array}$ & $\begin{array}{l}22 \\
26 \\
30\end{array}$ & $\begin{array}{l}91 / 6 \\
78 / 5\end{array}$ & 7 & 6 & $\begin{array}{l}2,250 \\
1,820 \\
1,900\end{array}$ & $\begin{array}{l}1,470 \\
1,420 \\
1,520\end{array}$ & $\begin{array}{l}610 \\
750 \\
820\end{array}$ & $\begin{array}{l}860 \\
670 \\
700\end{array}$ & $\begin{array}{l}1.8 \\
1.4 \\
1.7\end{array}$ & $\begin{array}{l}1.2 \\
1.0 \\
1.3\end{array}$ \\
\hline 17. E.W. & II (34) & $\begin{array}{r}104 / 60 \\
85 / 52\end{array}$ & $\begin{array}{l}76 \\
62\end{array}$ & $\begin{array}{l}86 / 42 \\
70 / 38\end{array}$ & $\begin{array}{l}56 \\
50\end{array}$ & $\begin{array}{l}24 \\
23\end{array}$ & $\begin{array}{l}32 \\
27\end{array}$ & $70 / 7$ & 5 & 2 & $\begin{array}{l}2,020 \\
1,320\end{array}$ & $\begin{array}{l}1,490 \\
1,060\end{array}$ & $\begin{array}{l}850 \\
570\end{array}$ & & $\begin{array}{l}2.0 \\
2.0\end{array}$ & $\begin{array}{l}1.4 \\
1.6\end{array}$ \\
\hline 18. A. K. & 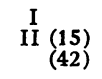 & $\begin{array}{r}123 / 72 \\
85 / 46 \\
85 / 44\end{array}$ & $\begin{array}{l}90 \\
60 \\
55\end{array}$ & $\begin{array}{r}105 / 38 \\
86 / 38 \\
69 / 33\end{array}$ & $\begin{array}{l}61 \\
55 \\
44\end{array}$ & $\begin{array}{l}35 \\
24 \\
18\end{array}$ & $\begin{array}{l}26 \\
31 \\
26\end{array}$ & $50 / 7$ & 6 & 5 & $\begin{array}{l}2,370 \\
1,340 \\
1,440\end{array}$ & $\begin{array}{l}1,550 \\
1,230 \\
1,150\end{array}$ & $\begin{array}{l}660 \\
680 \\
670\end{array}$ & $\begin{array}{l}890 \\
550 \\
480\end{array}$ & $\begin{array}{l}2.7 \\
2.0 \\
1.6\end{array}$ & $\begin{array}{l}1.8 \\
1.8 \\
1.2\end{array}$ \\
\hline 19. R. K. & II (30) & $\begin{array}{r}118 / 64 \\
98 / 54\end{array}$ & $\begin{array}{l}82 \\
65\end{array}$ & $\begin{array}{r}100 / 36 \\
78 / 33\end{array}$ & $\begin{array}{l}56 \\
47\end{array}$ & 25 & 29 & $67 / 7$ & 9 & 6 & $\begin{array}{l}2,370 \\
1,570\end{array}$ & $\begin{array}{l}1,620 \\
1,140\end{array}$ & 900 & 720 & $\begin{array}{l}1.7 \\
1.6\end{array}$ & $\begin{array}{l}1.0 \\
1.0\end{array}$ \\
\hline 20. H. J. & II (50) & $\begin{array}{l}158 / 84 \\
141 / 72\end{array}$ & $\begin{array}{r}100 \\
85\end{array}$ & $\begin{array}{r}113 / 45 \\
78 / 34\end{array}$ & $\begin{array}{l}66 \\
44\end{array}$ & $\begin{array}{l}30 \\
21\end{array}$ & $\begin{array}{l}36 \\
23\end{array}$ & $77 / 9$ & 9 & & $\begin{array}{l}2,560 \\
1,740\end{array}$ & $\begin{array}{r}1,690 \\
900\end{array}$ & $\begin{array}{l}920 \\
430\end{array}$ & $\begin{array}{l}770 \\
470\end{array}$ & $\begin{array}{l}2.9 \\
3.0\end{array}$ & $\begin{array}{l}1.8 \\
1.3\end{array}$ \\
\hline 21. E. J. & II $\underset{(64)}{(28)}$ & $\begin{array}{r}121 / 79 \\
78 / 51 \\
94 / 54\end{array}$ & $\begin{array}{l}98 \\
60 \\
66\end{array}$ & $\begin{array}{l}80 / 44 \\
66 / 30 \\
68 / 30\end{array}$ & $\begin{array}{l}55 \\
40 \\
41\end{array}$ & $\begin{array}{l}30 \\
22 \\
21\end{array}$ & $\begin{array}{l}25 \\
18 \\
20\end{array}$ & $\begin{array}{l}79 / 9 \\
63 / 6\end{array}$ & $\begin{array}{r}10 \\
7\end{array}$ & & $\begin{array}{l}3,650 \\
2,000 \\
2,060\end{array}$ & $\begin{array}{l}2,160 \\
1,320 \\
1,260\end{array}$ & $\begin{array}{l}940 \\
600 \\
620\end{array}$ & $\begin{array}{r}1,220 \\
720 \\
640\end{array}$ & $\begin{array}{l}1.9 \\
1.3 \\
1.6\end{array}$ & $\begin{array}{l}1.0 \\
0.7 \\
0.9\end{array}$ \\
\hline 22. B. $\mathrm{K}$. & II (30) & $\begin{array}{r}108 / 74 \\
89 / 59\end{array}$ & $\begin{array}{l}87 \\
68\end{array}$ & $\begin{array}{l}126 / 61 \\
103 / 41\end{array}$ & $\begin{array}{l}86 \\
61\end{array}$ & & & $95 / 11$ & 10 & 4 & $\begin{array}{l}3,370 \\
2,640\end{array}$ & $\begin{array}{l}3,320 \\
2,360\end{array}$ & & & $\begin{array}{l}1.4 \\
1.1\end{array}$ & $\begin{array}{l}1.3 \\
0.9\end{array}$ \\
\hline 23. A. C. & II ${ }_{\text {II }}^{\text {(47) }}$ & $\begin{array}{r}127 / 74 \\
74 / 43\end{array}$ & $\begin{array}{l}88 \\
53\end{array}$ & $\begin{array}{l}84 / 33 \\
49 / 25\end{array}$ & $\begin{array}{l}47 \\
35\end{array}$ & & & $\begin{array}{l}72 / 5 \\
49 / 4\end{array}$ & 3 & 2 & $\begin{array}{l}1,500 \\
1,040\end{array}$ & $\begin{array}{l}800 \\
690\end{array}$ & & & $\begin{array}{l}2.9 \\
1.6\end{array}$ & $\begin{array}{l}1.5 \\
1.0\end{array}$ \\
\hline 24. M. Mc. & II (39) & $\begin{array}{l}150 / 75 \\
138 / 74\end{array}$ & $\begin{array}{r}106 \\
94\end{array}$ & $\begin{array}{l}38 / 19 \\
37 / 18\end{array}$ & $\begin{array}{l}26 \\
25\end{array}$ & $\begin{array}{l}18 \\
14\end{array}$ & $\begin{array}{r}8 \\
11\end{array}$ & $37 / 3$ & 2 & 4 & $\begin{array}{l}2,620 \\
3,350\end{array}$ & $\begin{array}{l}640 \\
900\end{array}$ & $\begin{array}{l}190 \\
470\end{array}$ & $\begin{array}{l}450 \\
430\end{array}$ & $\begin{array}{l}3.1 \\
2.2\end{array}$ & $\begin{array}{l}0.7 \\
0.5\end{array}$ \\
\hline 25. G. A. & II (29) & $\begin{array}{l}166 / 81 \\
108 / 55\end{array}$ & $\begin{array}{r}114 \\
75\end{array}$ & $\begin{array}{l}61 / 31 \\
42 / 23\end{array}$ & $\begin{array}{l}42 \\
29\end{array}$ & $\begin{array}{l}27 \\
13\end{array}$ & $\begin{array}{l}15 \\
16\end{array}$ & $41 / 3$ & 2 & 1 & $\begin{array}{l}2,750 \\
1,920\end{array}$ & $\begin{array}{r}1,000 \\
740\end{array}$ & $\begin{array}{l}360 \\
410\end{array}$ & $\begin{array}{l}640 \\
330\end{array}$ & $\begin{array}{l}3.1 \\
2.0\end{array}$ & $\begin{array}{l}1.6 \\
0.8\end{array}$ \\
\hline 26. D. R. & II (29) & $\begin{array}{r}122 / 70 \\
88 / 50\end{array}$ & $\begin{array}{l}95 \\
63\end{array}$ & $\begin{array}{l}50 / 28 \\
36 / 17 \\
37 / 16\end{array}$ & $\begin{array}{l}35 \\
23 \\
23\end{array}$ & $\begin{array}{l}22 \\
14 \\
14\end{array}$ & $\begin{array}{r}13 \\
9 \\
9\end{array}$ & $35 / 4$ & 6 & 5 & $\begin{array}{l}1,230 \\
1,170\end{array}$ & $\begin{array}{r}450 \\
460 \\
430\end{array}$ & $\begin{array}{l}170 \\
180 \\
170\end{array}$ & $\begin{array}{l}280 \\
280 \\
260\end{array}$ & $\begin{array}{l}4.3 \\
1.9\end{array}$ & $\begin{array}{l}1.4 \\
0.6 \\
0.6\end{array}$ \\
\hline 27. J. Sc. & II (35) & $\begin{array}{r}114 / 81 \\
94 / 54\end{array}$ & $\begin{array}{l}92 \\
69\end{array}$ & $\begin{array}{l}86 / 44 \\
82 / 44\end{array}$ & $\begin{array}{l}63 \\
60\end{array}$ & 36 & 27 & $82 / 3$ & $\begin{array}{l}7 \\
3\end{array}$ & & $\begin{array}{l}1,900 \\
1,010\end{array}$ & $\begin{array}{r}1,250 \\
880\end{array}$ & 510 & 740 & $\begin{array}{l}2.9 \\
2.9\end{array}$ & $\begin{array}{l}1.8 \\
2.6\end{array}$ \\
\hline
\end{tabular}

F.A. = Systemic artery pressure.

"P.C." = Pulmonary artery pressure.

P.A.-"P.C." = Pulmonary artery to pulmonary "capillary" pressure gradient.

R.V. = Right ventricular pressure.
R.A. = Right atrial pressure.
L.V. = Left ventricular pressure (obtained at operation). $\mathrm{S}=$ Systolic; $\mathrm{D}=$ Diastolic; $\mathrm{M}=$ Mean.

$\begin{aligned} T S R & =\text { Total systemic resistance. } \\ \text { TPR } & =\text { Total pulmonary resistance } .\end{aligned}$

PVR = Pulmonary vascular resistance. 
patients), hexamethonium bromide (Hexameton $\left.{ }^{(B)}\right)^{\circ}$ diluted in physiological saline was injected through the catheter at a rate of about $1 \mathrm{mg}$. of ion per minute. Injection was stopped when the brachial artery systolic pressure, measured at one minute intervals with a sphygmomanometer, had fallen 30 to $40 \mathrm{~mm}$. $\mathrm{Hg}$. The total dose of ion was $2 \mathrm{mg}$. in 1 case, between 6 and 10 $\mathrm{mg}$. in 17 cases, and $11 \mathrm{mg}$. or more in the remaining 9 cases. In most cases the systemic blood pressure stabilized at the reduced level during the following 30 to 60 minutes. In a few patients there was a further drop in blood pressure one hour or more after the start of the injection. In two patients (D.C. and G.P.), the systemic blood pressure returned to the control level before the cardiac output determinations were repeated.

In all but one patient (M.B.), one or more determinations of cardiac output were made following hexamethonium injection, after which pulmonary artery and femoral artery pressures were again recorded in each case. Pulmonary "capillary" pressures were obtained in 20 cases. The respective data obtained were used for comparison and statistical analysis.

In any hemodynamic study there are limits of variation for each determinant. These have been studied in our laboratory and some of the findings reported previously $(14,18)$. In addition, studies were made on a series of 20 patients with two successive control determinations of systemic artery pressure, total systemic resistance, left and right ventricular works against pres-

6 Courtesy of Burroughs Wellcome \& Co., Inc., Tuckahoe, N. Y. sure. A summary of the ranges of variation of all the determinants for the repeated measurements in the same patient is given below.

Cardiac index (C.I.)

Stroke index (S.I.)

Systemic artery mean pressure $\left(F_{m}\right)$

Pulmonary artery mean pressure $\left(\mathrm{PA}_{\mathrm{m}}\right)$

Pulmonary "capillary" mean pressure ("PC"'m)

$\mathrm{PA}_{m}$ - "PC"'m gradient

$\left(\mathrm{PA}_{\mathrm{m}}-\right.$ "PC"m)

Total systemic resistance (TSR)

Total pulmonary resistance (TPR)

Pulmonary vascular resistance (PVR)

"Left heart resistance" ("LHR")

Left ventricular work against $\quad \pm 25$ per cent pressure (LVW)

Right ventricular work against \pm 25 per cent pressure (RVW)

Deviations of individual results beyond these limits are referred to hereafter as "individually significant" in-

TABLE II

Statistical data in hemodynamic changes following hexamethonium injection *

\begin{tabular}{|c|c|c|c|c|c|c|}
\hline & $\begin{array}{l}\text { No. of } \\
\text { cases }\end{array}$ & $\underset{\text { mean }}{\text { Control }}$ & $\begin{array}{l}\text { Hexamethonium } \\
\text { mean }\end{array}$ & $\begin{array}{l}\text { Standard } \\
\text { error of } \\
\text { difference }\end{array}$ & $\begin{array}{l}\text { Difference as } \\
\text { per cent of } \\
\text { control } \\
\text { mean }\end{array}$ & pt \\
\hline $\begin{array}{l}\text { Blood flow } \\
\text { C.I. } \\
\text { S.I. } \\
\text { H.R. }\end{array}$ & $\begin{array}{l}26 \\
26 \\
27\end{array}$ & $\begin{array}{l}2.39 \\
27.4 \\
98\end{array}$ & $\begin{array}{l}2.44 \\
28.2 \\
98\end{array}$ & $\begin{array}{l}0.06 \\
1.32 \\
2.11\end{array}$ & $\begin{array}{r}+2 \\
+3 \\
0\end{array}$ & $\begin{array}{l}>0.1 \\
>0.1 \\
>0.1\end{array}$ \\
\hline \multicolumn{7}{|l|}{ Blood pressures } \\
\hline $\begin{array}{l}\mathrm{FA}_{m} \\
\mathrm{PA}_{\mathrm{m}} \\
\text { "PC" } \\
\mathrm{PA}_{\mathrm{m}}-" \mathrm{PC} "{ }_{\mathrm{m}}\end{array}$ & $\begin{array}{l}26 \\
27 \\
20 \\
20\end{array}$ & $\begin{array}{l}91.9 \\
46.9 \\
28.9 \\
18.3\end{array}$ & $\begin{array}{l}72.6 \\
37.7 \\
22.0 \\
16.0\end{array}$ & $\begin{array}{l}2.67 \\
1.15 \\
1.29 \\
1.14\end{array}$ & $\begin{array}{l}-21.0 \\
-18.5 \\
-24.1 \\
-12.6\end{array}$ & $\begin{array}{l}<0.005 \\
<0.005 \\
<0.005 \\
\quad 0.05<\mathrm{p}<0.1\end{array}$ \\
\hline \multicolumn{7}{|l|}{ Resistances } \\
\hline $\begin{array}{l}\text { TSR } \\
\text { TPR } \\
\text { PVR } \\
\text { "LHR" }\end{array}$ & $\begin{array}{l}26 \\
26 \\
19 \\
19\end{array}$ & $\begin{array}{r}2,042 \\
1,128 \\
434 \\
596\end{array}$ & $\begin{array}{r}1,594 \\
868 \\
370 \\
419\end{array}$ & $\begin{array}{l}9.32 \\
5.52 \\
4.03 \\
3.43\end{array}$ & $\begin{array}{l}-21.9 \\
-31.9 \\
-14.7 \\
-29.7\end{array}$ & $\begin{array}{l}<0.005 \\
<0.005 \\
>0.1 \\
<0.005\end{array}$ \\
\hline \multicolumn{7}{|c|}{$\begin{array}{l}\text { Ventricular work } \\
\text { against pressure }\end{array}$} \\
\hline $\begin{array}{l}\text { LVW } \\
\text { RVW }\end{array}$ & $\begin{array}{l}20 \\
26\end{array}$ & $\begin{array}{l}2.78 \\
1.42\end{array}$ & $\begin{array}{l}2.19 \\
1.19\end{array}$ & $\begin{array}{l}0.152 \\
0.072\end{array}$ & $\begin{array}{l}-21.2 \\
-16.3\end{array}$ & $\begin{array}{l}<0.005 \\
<0.005\end{array}$ \\
\hline
\end{tabular}

* The abbreviations and units in this table are identical to those used in Table I.

t The symbol of "p" indicates the probability that a difference as large as that observed will occur by chance. Throughout the discussion, a difference with a chance probability of 0.05 or less is considered to be significant. 
TABLE III

Individually significant differences in the hemodynamic determinants following hexamethonium injection *

\begin{tabular}{|c|c|c|c|}
\hline & \multirow[b]{2}{*}{ Total } & \multicolumn{2}{|c|}{ No. of cases } \\
\hline & & $\begin{array}{l}\text { showing } \\
\text { individually } \\
\text { significant } \\
\text { increase }\end{array}$ & $\begin{array}{l}\text { showing } \\
\text { individually } \\
\text { significant } \\
\text { decrease }\end{array}$ \\
\hline \multicolumn{4}{|l|}{ Blood flow } \\
\hline $\begin{array}{l}\text { C.I. } \\
\text { S.I. }\end{array}$ & $\begin{array}{l}26 \\
25\end{array}$ & $\begin{array}{l}7 \\
4\end{array}$ & $\begin{array}{l}4 \\
4\end{array}$ \\
\hline \multicolumn{4}{|l|}{ Pressures } \\
\hline $\begin{array}{l}\mathrm{FA}_{m} \\
\mathrm{PA}_{\mathbf{m}} \\
\text { "PC" } \\
\mathrm{PA}_{m}-" \mathrm{PC} "{ }_{m}\end{array}$ & $\begin{array}{l}26 \\
25 \\
20 \\
20\end{array}$ & $\begin{array}{l}0 \\
0 \\
1 \\
2\end{array}$ & $\begin{array}{r}19 \\
14 \\
14 \\
5\end{array}$ \\
\hline \multicolumn{4}{|l|}{ Resistances } \\
\hline $\begin{array}{l}\text { TSR } \\
\text { TPR } \\
\text { PVR } \\
\text { "LHR" }\end{array}$ & $\begin{array}{l}26 \\
26 \\
19 \\
19\end{array}$ & $\begin{array}{l}2 \\
1 \\
3 \\
0\end{array}$ & $\begin{array}{r}14 \\
12 . \\
6 \\
\cdot 14\end{array}$ \\
\hline \multicolumn{4}{|c|}{$\begin{array}{l}\text { Ventricular work } \\
\text { against pressure }\end{array}$} \\
\hline $\begin{array}{l}\text { LVW } \\
\text { RVW }\end{array}$ & $\begin{array}{l}20 \\
26\end{array}$ & $\begin{array}{l}0 \\
2\end{array}$ & $\begin{array}{l}12 \\
12\end{array}$ \\
\hline
\end{tabular}

* The abbreviations and units in this table are identical to those used in Table I.

creases or decreases. The term "statistically significant" refers to differences between two means having a " $\mathrm{p}$ " value less than 0.05 .

A few days or weeks following the cardiac catheterization, mitral valvuloplasty was performed by Dr. E. B. Mahoney on all but three patients (M.B., H.J., and J.Sc.). At operation, atrial and left ventricular pressures were recorded immediately before and after fracture of the mitral valve in 23 cases. A pressure gradient of $6 \mathrm{~mm}$. $\mathrm{Hg}$ or more across the mitral valve during diastole was found in all but one patient (R.B.). The left ventricular diastolic pressure was $8 \mathrm{~mm}$. $\mathrm{Hg}$ or less in 18 of the 23 cases. In the remaining five cases, the left ventricular diastolic pressures were $9,12,13,14$ and $16 \mathrm{~mm}$. $\mathrm{Hg}$ and suggested the presence of concomitant left ventricular failure.

\section{RESULTS}

The results are given in detail in Table $I$ and summarized in Tables II and III. It will be seen from Table II that following hexamethonium injection there were statistically significant decreases in femoral artery pressure, pulmonary artery pressure, and pulmonary "capillary" pressure, but no statistically significant changes in cardiac index, stroke index, heart rate, pulmonary artery to pulmonary "capillary" pressure gradient and pulmonary vascular resistance. The total systemic, total pulmonary, "left heart" resistances (Figure 1 ), and the work of each ventricle against pressure were all statistically significantly reduced. The incidence of individually significant changes in these determinants is given in Table III.

The decrease in pulmonary artery pressure usually occurred within 10 minutes of the start of the injection, and at the same time as, or shortly following, the fall in femoral artery pressure. The systolic pressure in the pulmonary artery was more affected than the diastolic. The fall in mean pres-

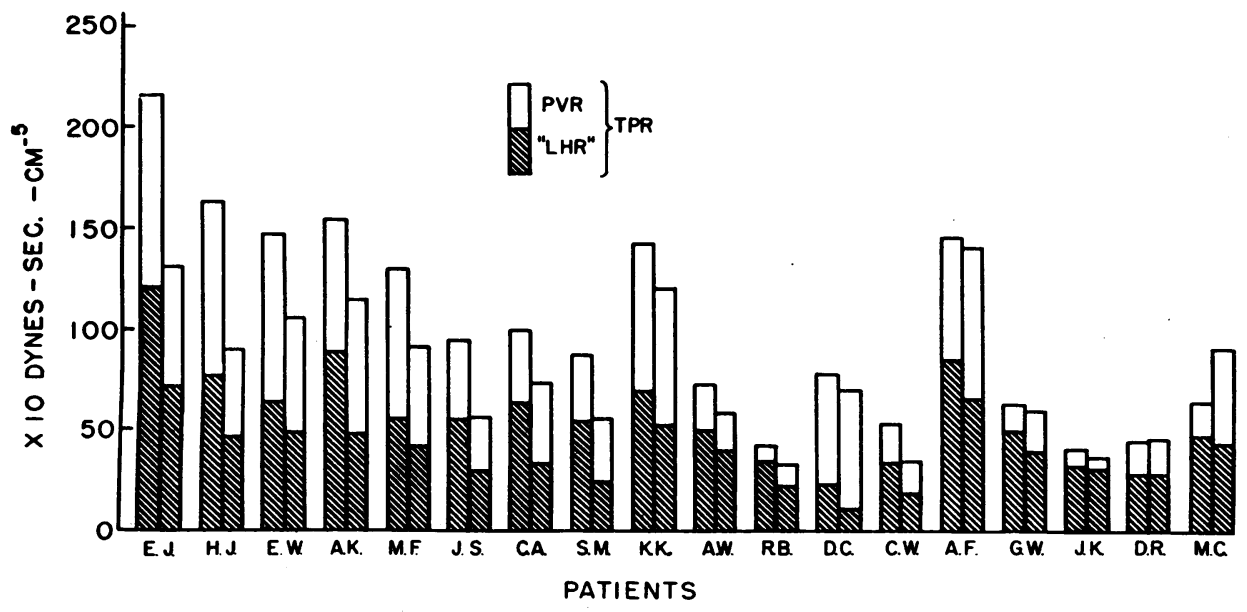

Fig. 1. Change in Total Pulmonary, Pulmonary Vascular and "Left Heart" Resistances Following Hexamethonium Injection in 18 Patients with Mitral Stenosis

The first column of each pair indicates the resistances measured during the control period and the second column shows the resistances after hexamethonium injection. 


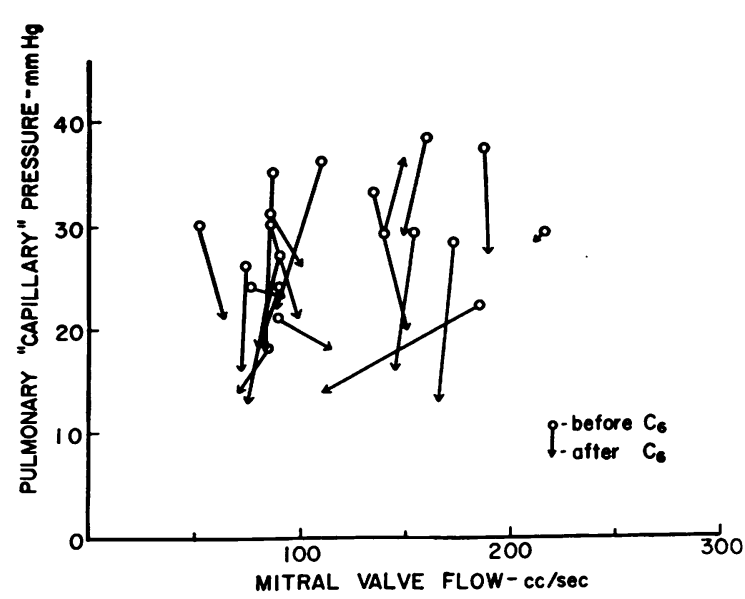

Fig. 2. Relation Between Pulmonary "Capillary" Pressure and Mitral Valve Flow Before and After Hexamethonium

The pressure changes are unrelated to the trivial alterations in flow.

sure was, in most cases, proportionally similar in the pulmonary and femoral arteries.

The decrease in pulmonary "capillary" pressure was also roughly proportional, in most cases, to the decrease in the femoral artery pressure. The change in pulmonary "capillary" pressure was unrelated to changes in flow, whether the latter was expressed per minute, per heart beat, or per second of diastolic time (Figure 2). It was also unrelated to the change in pulmonary vascular resistance. The "capillary" pressure changes were significantly positively correlated with the changes in "left heart resistance," left ventricular work against pressure, and right ventricular work against pressure.

\section{Use of intravenous hexamethonium in pulmonary edema}

Intravenous hexamethonium was used clinically with excellent response in treating acute pulmonary edema in three patients with mitral stenosis. In two additional patients with mitral stenosis, acute pulmonary edema which developed during cardiac catheterization was promptly controlled after the injection of hexamethonium through the catheter. In the first case the pulmonary artery pressure rose from a control value of $124 / 65$ (mean, 84) to $140 / 100 \mathrm{~mm}$. $\mathrm{Hg}$ (mean, 113) during acute pulmonary edema. A total of
$10 \mathrm{mg}$. of hexamethonium ion was given over a period of 15 minutes, during which time the pulmonary artery pressure decreased to $92 / 44 \mathrm{~mm}$. $\mathrm{Hg}$ (mean, 58). In the other case, the pulmonary artery pressure was $72 / 38 \mathrm{~mm}$. $\mathrm{Hg}$ (mean, 47) at rest, rose to $130 / 70 \mathrm{~mm} . \mathrm{Hg}$ during acute pulmonary edema, and decreased to $51 / 27 \mathrm{~mm}$. $\mathrm{Hg}$ (mean, 35) 20 minutes after injection of $12 \mathrm{mg}$. of hexamethonium ion. The details of these observations will be reported in a separate communication.

\section{DISCUSSION}

Our results are in agreement with several reports that ganglionic blocking agents, given to patients with mitral stenosis, reduce the pulmonary artery and pulmonary "capillary" pressures without significantly altering the flow $(5,9,10,19)$. This pattern is different from that commonly observed in normal animals $(20,21)$, in patients without cardiorespiratory disease (4), and in a variety of cardiorespiratory diseases other than mitral stenosis $(1,6,8,22)$. In these groups, cardiac output usually falls sufficiently to account for the decline in pulmonary and systemic pressures without invoking changes in resistance. This type of response, ascribed to peripheral pooling, was found by Storstein and Tveten (6) to occur also in eight patients with mitral stenosis. We have no explanation for the discrepancy between their results in this disease and those of others including ourselves.

The paradox of reduced pulmonary "capillary" pressure without change in flow has not, to our knowledge, been satisfactorily explained. The explanation cannot lie simply in vasomotor changes in any part of the lung, nor in passive shifts of blood to the periphery, for any change in left atrial pressure so induced would of necessity change the flow also.

There are two possible explanations which seem to us most likely to be correct: 1) a drop in left ventricular diastolic pressure, and 2) intrapulmonary shunting of blood.

Only five of our patients were found later to have abnormally high left ventricular diastolic pressures, and these were not those who had the most marked drops of pulmonary "capillary" pressure at catheterization.

Nevertheless, a decline of this pressure follow- 
ing hexamethonium has been noted in normal dogs (19) and it would, of course, permit a similar drop to occur in the left atrial pressure without reducing the flow.

Intrapulmonary shunts connecting the pulmonary vein to the azygos vein and right atrium by way of varicose anastomoses with the bronchial vein are known to exist in mitral stenosis (23). When the pulmonary veins of one lung are ligated in dogs, such anastomoses may carry up to 20 per cent of the normal flow for that lung, so that azygos vein blood resembles arterial blood (24). If such anastomoses in mitral stenosis patients were subject to dilation by hexamethonium, as systemic veins appear to be, then the paradox could be explained, as would the difference in response between mitral stenosis and other diseases. The increased low-pressure runoff would probably lower pulmonary venous pressure, which in turn would reduce the flow through the mitral valve. The shunted arterialized blood would recirculate through the lungs. The change in pulmonary flow, as we measured it, might therefore be nil, or even an increase, depending on the balance between increase in shunt flow and decrease in mitral valve flow.

Among other explanations we have discarded an actual reduction of mitral valve resistance as highly unlikely on structural grounds. It is true that marked slowing of the heart rate was associated with the largest decrease in pulmonary "capillary" pressure per unit flow. However, the overall change in heart rate was insignificant, and even when the length of diastole is taken into account, there were no flow changes to correlate with the alterations in pressure (Figure 2). Lastly, it seems unlikely that we are dealing with vasomotor changes in the recently postulated zone of resistance in the pulmonary venous bed (25).

The decline in pulmonary vascular resistance, which was noted in six cases, it not likely to result passively from the lowering of left atrial pressure, since this should have the opposite effect (26). Neither does it seem likely to be due to a vasodilator reflex from pressure receptors in the pulmonary capillary-venous bed (9), since the changes were unrelated to changes in pulmonary "capillary" pressure. It is possible that it was caused by ganglionic blockade of the pulmonary arteriolar bed $(2,5,19)$.

\section{SUMMARY}

1. Hexamethonium bromide was administered through a catheter into the pulmonary artery of 27 patients with mitral stenosis in doses sufficient to produce an initial depression of about $30 \mathrm{~mm} . \mathrm{Hg}$ in the systolic pressure in the brachial artery.

2. Over the course of the following half-hour to one hour, changes in cardiac output, heart rate and stroke volume were insignificant.

3. The pulmonary artery pressure declined significantly in over half the cases, and the change was roughly proportional to the change in systemic artery pressure. The pulmonary "capillary" pressure also fell in more than two-thirds of observations. There was no consistent change in the $\mathrm{PA}_{\mathrm{m}}-$ "PC" $\mathrm{m}$ gradient, although pulmonary vascular resistance fell in a few instances.

4. The paradox of declining pulmonary "capillary" pressure with constant cardiac output is discussed. On hydraulic grounds it is unlikely that this could be explained solely by any vasomotor changes in the lung vessels or by passive shifts of blood from pulmonary to peripheral reservoirs, although both these may occur in addition. Changes in heart rate may play a small contributing role. Two other possible explanations are discussed: changes in left ventricular diastolic pressure, and increases in the transport of blood through intrapulmonary vascular shunts.

5. The clinical importance of the action of hexamethonium on the pressure in pulmonary circuit is illustrated by two cases of its successful use in the treatment of acute pulmonary edema which developed in patients with mitral stenosis during cardiac catheterization.

\section{ACKNOWLEDGMENT}

We wish to express our thanks and appreciation to Dr. S. Lee Crump, Associate Professor of Radiation Biology and Scientist (Statistics), Atomic Energy Project, University of Rochester, for his help on the statistical aspects of this study. We are indebted to Mrs. Ruth Knight for technical assistance, and to Miss Irene Subrani for preparing the manuscript.

\section{REFERENCES}

1. Werkö, L., Frisk, A. R., Wade, G., and Eliasch, H. Effect of hexamethonium bromide in arterial hypertension. Lancet 1951, 2, 470.

2. Gilmore, H. R., Kopelman, H., McMichael, J., and Milne, I. G. The effect of hexamethonium bro- 
mide on the cardiac output and pulmonary circulation. Lancet 1952, 2, 898.

3. Kelley, R. T., Freis, E. D., and Higgins, T. F. The effects of hexamethonium on certain manifestations of congestive heart failure. Circulation 1953, 7, 169.

4. Rakita, L., and Sancetta, S. M. Acute hemodynamic effects of hexamethonium in normotensive man. Circ. Res. 1953, 1, 499.

5. Davies, L. G., Goodwin, J. F., and VanLeuven, B. D. The nature of pulmonary hypertension in mitral stenosis. Brit. Heart J. 1954, 16, 440.

6. Storstein, O., and Tveten, H. The effect of hexamethonium bromide on the pulmonary circulation. Scand. J. clin. Lab. Invest. 1954, 6, 169.

7. Burch, R. R. The effects of intravenous hexamethonium on venous pressure of normotensive and hypertensive patients with and without congestive heart failure. Circulation 1955, 11, 271.

8. Sancetta, S. M. Acute hemodynamic effects of hexamethonium $\left(\mathrm{C}_{6}\right)$ in patients with emphysematous pulmonary hypertension. Amer. Heart J. 1955, 49, 501.

9. Wade, E. G., Mackinnon, J., and Vickers, C. F. H. The nature of the increased pulmonary vascular resistance in mitral stenosis. Brit. Heart J. 1956, $18,458$.

10. Balchum, O. J., Gensini, G., and Blount, S. G., Jr. The effect of hexamethonium upon the pulmonary vascular resistance in mitral stenosis. J. Lab. clin. Med. 1957, 50, 186.

11. Yu, P. N., Lovejoy, F. W., Jr., Joos, H. A., Nye, R. E., Jr., and McCann, W. S. Studies of pulmonary hypertension. I. Pulmonary circulatory dynamics in patients with pulmonary emphysema at rest. J. clin. Invest. 1953, 32, 130.

12. Yu, P. N., Simpson, J. H., Lovejoy, F. W., Jr., Joos, H. A., and Nye, R. E., Jr. Studies of pulmonary hypertension. IV. Pulmonary circulatory dynamics in patients with mitral stenosis at rest. Amer. Heart J. 1954, 47, 330.

13. Yu, P. N., Lovejoy, F. W., Jr., Joos, H. A., Nye, R. E., Jr., Beatty, D. C., and Simpson, J. H. Studies of pulmonary hypertension. VI. Pulmonary "capillary" pressure in various cardiopulmonary diseases at rest and under stress. Amer. Heart J. 1955, 49, 31.

14. Yu, P. N., Beatty, D. C., Lovejoy, F. W., Jr., Nye, R. E., Jr., and Joos, H. A. Studies of pulmonary hypertension. VII. Hemodynamic effects of acute hypoxia in patients with mitral stenosis. Amer. Heart J. 1956, 52, 683.
15. Gorlin, R., and Gorlin, S. G. Hydraulic formula for calculation of the area of the stenotic mitral valve, other cardiac valves, and central circulation shunts. Amer. Heart J. 1951, 41, 1.

16. Gorlin, R., Haynes, F. W., Goodale, W. T., Sawyer, C. G., Dow, J. W., and Dexter, L. Studies of the circulatory dynamics in mitral stenonsis. II. Altered dynamics at rest. Amer. Heart. J. 1951, 41, 30.

17. Werkö, L., Biörck, G., Crafoord, C., Wulff, H., Krook, H., and Eliasch, H. Pulmonary circulatory dynamics in mitral stenosis before and after commissurotomy. Amer. Heart J. 1953, 45, 477.

18. Yu, P. N., Nye, R. E., Jr., Lovejoy, F. W., Jr., Macias, J. deJ., Schreiner, B. F., and Lux, J. J. Studies of pulmonary hypertension. VIII. The effects of acetyl strophanthidin on pulmonary circulation in patients with cardiac failure and mitral stenosis. Amer. Heart J. 1957, 54, 235.

19. Scott, R. C., Kaplan, S., and Stiles, W. J. Observations on the effect of tetraethylammonium chloride on the pulmonary vascular resistance in mitral stenosis. Amer. Heart J. 1955, 50, 720.

20. Wakim, K. G. Certain cardiovasculorenal effects of hexamethonium. Amer. Heart J. 1955, 50, 435.

21. Combes, B., Preedy, J. R. K., Wheeler, H. O., Hays, R. M., and Bradley, S. E. The hemodynamic effects of hexamethonium bromide in the dog, with special reference to "splanchnic pooling." J. clin. Invest. 1957, 36, 860.

22. Smith, J. R., and Hoobler, S. W. Acute and chronic cardiovascular effects of pentolinium in hypertensive patients. Circulation 1956, 14, 1061.

23. Ferguson, F. C., Gobilak, R. E., and Deitrick, J. E. Varices of the bronchial veins as a source of hemoptysis in mitral stenosis. Amer. Heart J. 1944, 28, 445.

24. Hurwitz, A., Calabresi, M., Cooke, R. W., and Liebow, A. A. An experimental study of the venous collateral circulation of the lung. II. Functional observations. J. thorac. Surg. 1954, 28, 241.

25. Tosetti, R. Les zones de résistance vasulaire pulmonaire au cours du retrécissement mitral et du coeur pulmonaire chroniquedos emphysémateux; étude de leur localisation. Arch. Mal. Coeur, 1955, 48, 346. (Abstract in Circulation 1956, 13, 784.)

26. Borst, H. G., McGregor, M., Whittenberger, J. L., and Berglund, E. Influence of pulmonary arterial and left atrial pressures on pulmonary vascular resistance. Circ. Res. 1956, 4, 393. 